\title{
Supersingular Isogeny Oblivious Transfer
}

\author{
Paulo Barreto $^{1}$, Gláucio Oliveira ${ }^{2}$, Waldyr Benits ${ }^{3}$, Anderson Nascimento ${ }^{1}$ \\ ${ }^{1}$ Escola de Engenharia \& Tecnologia - Universidade de Washington, Tacoma, EUA. \\ ${ }^{2}$ Instituto de Matemática e Estatística - Universidade de São Paulo (IME-USP) \\ ${ }^{3}$ Centro de Análises de Sistemas Navais - Marinha do Brasil (CASNAV-MB) \\ \{pbarreto, andcla\}@uw.edu, glaucioaorj@gmail.com, wbenits@yahoo.com.br
}

\begin{abstract}
In this paper we present an Oblivious Transfer (OT) protocol that combines an OT scheme together with the supersingular isogeny Diffie-Hellman (SIDH) primitive. Our proposal is a candidate for post-quantum secure OT and demonstrates that SIDH naturally supports OT functionality. We consider the protocol in the simplest configuration of $\left(\begin{array}{l}2 \\ 1\end{array}\right)$-SIOT and analyze the protocol to verify its security.
\end{abstract}

Resumo. Neste artigo, apresentamos um protocolo Oblivious Transfer (OT) que combina um esquema OT juntamente com a primitiva do protocolo Supersingular Isogeny Diffie-Hellman (SIDH). Nossa proposta é um candidato para segurança pós-quântica OT e demonstra que o SIDH naturalmente suporta a funcionalidade OT. Consideramos o protocolo na configuração mais simples de $\left(\begin{array}{l}2 \\ 1\end{array}\right)$-SIOT e analisamos a sua segurança.

\section{Introdução}

[Rabin 1981] propôs a primeira noção de Oblivious Transfer (OT). Desde então, muitos protocolos criptográficos foram implementados utilizando uma estrutura OT. [Chou and Orlandi 2015] afirmam que eficientes protocolos OT são vulneráveis em um cenário quântico, em que a hipótese de segurança se baseia na dificuldade de resolver o Problema do Logaritmo Discreto ou de fatorar números inteiros. Vários artigos tem introduzido o sistema OT no contexto da criptografia quântica tais como [Crepeau and Kilian 1988] e [Bennett et al. 1992] entre outros, onde os usuários legítimos manipulam estados quânticos. Por outro lado, a pesquisa em construções OT pós-quânticas tem crescido gradualmente. Desta forma, pode-se citar, por exemplo, os trabalhos de [Kazmi 2015], e [Vitse 2019]. Em geral, em um protocolo $\left(\begin{array}{l}2 \\ 1\end{array}\right)$-OT, o remetente envia duas mensagens distintas, $m_{a}$ e $m_{b}$ para um destinatário, que escolhe somente uma delas para ter acesso ao conteúdo. No final do protocolo, o remetente não tem o conhecimento de qual mensagem foi escolhida e, também, o destinatário não tem acesso ao conteúdo da outra mensagem.

Nossa contribuição. De acordo com [Hazay and Lindell. 2010], OT é uma das estruturas mais importantes em criptografia e vantajosa para a construção de protocolos seguros. Em termos de aplicação, esses tipos de protocolos podem ser utilizados em processos de leilões eletrônicos, ou ainda em assinaturas de contratos [Even et al. 1985] ou esquemas de transações com dinheiro eletrônico [Barak 2007]. Neste trabalho, o nosso objetivo principal foi a implementação do $\left(\begin{array}{l}2 \\ 1\end{array}\right)$-SIOT usando as primitivas do SIDH de [Feo et al. 2014] 
com o propósito de proporcionar privacidade entre remetente e destinatário e, ao mesmo tempo, prover uma resistência contra o eminente advento da computação quântica.

Este artigo está organizado da seguinte forma: A seção 2 descreve o protocolo $\left(\begin{array}{l}2 \\ 1\end{array}\right)$-SIOT bem como apresenta uma análise de segurança. Na seção 3 , apresentamos a conclusão da análise de segurança do protocolo proposto. A seção 4 mostra alguns resultados e parâmetros do $\left(\begin{array}{l}2 \\ 1\end{array}\right)$-SIOT. Por fim, na seção 5 , tem-se a conclusão.

\section{Protocolo $\left(\begin{array}{l}2 \\ 1\end{array}\right)$-SIOT}

\subsection{Notações}

Nós utilizamos as primitivas criptográficas do protocolo de troca de chaves SIDH de [Feo et al. 2014]. Assim, as seguintes notações serão usadas:

i. $\mathcal{M}, \mathcal{K}, \mathcal{C} \rightarrow$ Conjunto de todos os textos claros, chaves e cifras em uma cadeia binária de bits com comprimento fixado, respectivamente;

ii. $p \rightarrow$ um número primo tal que $p=3(\bmod 4)$;

iii. $\mathbb{F}_{p^{2}} \rightarrow$ Uma extensão quadrática de um corpo $\mathbb{F}_{p}$, onde $\mathbb{F}_{p^{2}}=\mathbb{F}_{p}[i] /\left\langle i^{2}+1\right\rangle$;

iv. $E_{0}\left[\mathbb{F}_{p^{2}}\right] \rightarrow$ Uma curva elíptica supersingular sobre $\mathbb{F}_{p^{2}}$;

v. $\mathbb{Z} / \ell \mathbb{Z} \rightarrow$ Um corpo de inteiros módulo $\ell$, onde $\ell$ é um número primo tal que $\ell \nmid p$;

vi. $P_{A}, Q_{A} \rightarrow$ Pontos linearmente independentes sobre uma curva elíptica supersingular $E_{0}\left[\ell_{A}^{e_{A}}\right]$

vii. $P_{B}, Q_{B} \rightarrow$ Pontos linearmente independentes sobre uma curva elíptica supersingular $E_{0}\left[\ell_{B}^{e_{B}}\right]$

viii. $\phi_{A}, \phi_{B} \rightarrow$ Isogenias entre as curvas elípticas supersingulares $E_{0}$ e $E_{A}, E_{0}$ e $E_{B}$, respectivamente;

ix. $\phi_{A}^{\prime}, \phi_{B}^{\prime} \rightarrow$ Isogenias entre as curvas elípticas supersingulares $E_{B}$ e $E_{B A}, E_{A}$ e $E_{A B}$, respectivamente;

x. $G_{A}, H_{A} \rightarrow$ Imagens dos pontos $P_{B}$ e $Q_{B}$ sobre a isogenia $\phi_{A}$ de um remetente;

xi. $G_{B}, H_{B} \rightarrow$ Imagens dos pontos $P_{A}$ e $Q_{A}$ sobre a isogenia $\phi_{B}$ de um destinatário;

xii. $\jmath\left(E_{A B}\right), \jmath\left(E_{B A}\right) \rightarrow$ Invariante $\jmath$ das curvas elípticas supersingulares $E_{A B}$ e $E_{B A}$, respectivamente;

xiii $e_{A}, e_{B} \rightarrow$ Números inteiros positivos;

xiv. $r_{A}, r_{B} \rightarrow$ Inteiros de $\mathbb{Z} / \ell_{A}^{e_{A}} \mathbb{Z} \mathrm{e} \mathbb{Z} / \ell_{B}^{e_{B}} \mathbb{Z}$, respectivamente;

xv. $f \rightarrow$ Um cofator para garantir um número primo da forma $p=\ell_{A}^{e_{A}} \ell_{B}^{e_{B}} f \pm 1$;

xvi. $\mathcal{H} \rightarrow$ Função hash;

xviii. $\mathcal{E}, \mathcal{D} \rightarrow$ Algoritmos de Encriptação e Decriptação, respectivamente;

xviii. $\mathrm{pk}_{A}, \mathrm{pk}_{B} \rightarrow$ Chaves públicas do remetente e destinatário, respectivamente;

xiv. $\mathrm{sk}_{A}, \mathrm{sk}_{B} \rightarrow$ Chaves privadas do remetente e destinatário, respectivamente;

xx. $\mathcal{O} \rightarrow$ Ponto especial localizado no infinito. Atua como um elemento neutro em operações de adição de pontos de uma curva elíptica sobre corpos finitos;

xxi. $\operatorname{ker}(\phi) \rightarrow$ Kernel de uma isogenia $\phi$. Em particular, é um subgrupo finito de uma curva elíptica $E$ sobre um corpo fechado $\overline{\mathbb{F}}_{p^{2}}$. Pode ser também denotado por $\langle P, Q\rangle$, onde os pontos $P, Q \in E\left[\overline{\mathbb{F}}_{p^{2}}\right]$. 


\subsection{Parâmetros Públicos}

Seja $E_{0}$ uma curva elíptica supersingular definida sobre um corpo $\mathbb{F}_{p^{2}}$. Por conveniência, suponha a escolha de um número primo da forma $p=\ell_{A}^{e_{A}} \ell_{B}^{e_{B}} f-1$ onde $\ell_{A}=2, e_{A} \geqslant 4$ e $f=1$ ou $p=4 \ell_{A}^{e_{A}} \ell_{B}^{e_{B}}-1$ onde $\ell_{A}$ e $\ell_{B}$ são primos ímpares e, $f=4$. Assim, qualquer uma dessas referidas escolhas resultam em um número primo da forma $p=3(\bmod 4)$. Isso habilita a representaçãd ${ }^{1} \mathbb{F}_{p^{2}}=\mathbb{F}_{p}[i] /\left(i^{2}+1\right)$ e assegura que a curva $E_{0}\left[\mathbb{F}_{p^{2}}\right]: y^{2}=$ $x^{3}+x$ seja supersingular com uma ordem de grupo igual $\left(\ell_{A}^{e_{A}} \ell_{B}^{e_{B}}\right)^{2}$. Adicionalmente, sejam $P_{A}, Q_{A} \in \mathbb{F}_{p^{2}}$ uma base de $E_{0}\left[\ell_{A}^{e_{A}}\right]$. De forma similar, outros pontos $P_{B}$ e $Q_{B}$ geram $E_{0}\left[\ell_{B}^{e_{B}}\right]$. Em outras palavras, $E_{0}\left[\ell_{A}^{e_{A}}\right]$ e $E_{0}\left[\ell_{B}^{e_{B}}\right]$ são gerados pelo kernel $\left\langle P_{A}, Q_{A}\right\rangle \mathrm{e}$ $\left\langle P_{B}, Q_{B}\right\rangle$, respectivamente.

\subsection{Premissas}

Em um canal de comunicação estabelecido no protocolo $\left(\begin{array}{l}2 \\ 1\end{array}\right)$-SIOT, o remetente e o destinatário serão usualmente chamados de Alice e Bob, respectivamente.

1. Seja um esquema de criptografia simétrica $(\mathcal{E}, \mathcal{D})$, de acordo com as definições 1 e 2 de [Chou and Orlandi 2015]. A chave simétrica compartilhada é o valor do invariante $\jmath$ entre duas curvas elípticas supersingulares e isomorfas $2^{2}$ Como será visto na figura 1, esse invariante $\jmath$ será submetido a uma função hash $\mathcal{H}=$ $\left\{H_{k}: k \in \mathcal{K}\right\}$ indexada por um conjunto finito $\mathcal{K}$, onde cada $H_{k}$ é uma função de $\mathbb{F}_{p^{2}}$

2. Alice deseja encriptar duas mensagens $m_{0}, m_{1} \in \mathcal{M}$ e enviá-las para Bob. Por sua vez, Bob irá decriptar somente uma dessas duas mensagens e Alice não terá conhecimento de sua escolha;

3. Alice e Bob utilizam um protocolo coin-flipping de [Wagner 2016] para compartilharem uma única cadeia aleatória e uniforme de bits $w$. Isso assegura que nem Alice e nem Bob podem adivinhar antecipadamente ou controlar o valor de $w$. Em seguida, eles devem utilizar, por exemplo, uma função hash nessa cadeia de bits para obter os pontos $U$ e $V$ linearmente independentes. Caso contrário, eles devem gerar uma nova cadeia $w$.

\subsection{Protocolo}

A figura 1 mostra uma abstração do funcionamento do protocolo proposto na sua forma mais simples, isto é, $\left(\begin{array}{l}2 \\ 1\end{array}\right)$ - SIOT.

\subsubsection{Geração de par de chaves}

\subsubsection{Setup - Remetente}

1. Alice escolhe secretamente um valor $r_{A} \in Z / \ell_{A}^{e_{A}} Z$;

2. Ela calcula:

(a) $\operatorname{ker}\left(\phi_{A}\right)=\left\langle P_{A}+r_{A} Q_{A}\right\rangle$;

(b) $\phi_{A}: E_{0} \rightarrow E_{A}$;

\footnotetext{
${ }^{1}$ Veja [Hoffstein et al. 2014], corolário 2.56.

${ }^{2}$ Veja [Silvermann 2009], proposição III.1.4 (b).
} 


\begin{tabular}{|c|c|c|}
\hline & \multicolumn{2}{|c|}{ Supersingular Isogeny Oblivious Transfer (SIOT) } \\
\hline & & Bob \\
\hline \multirow{13}{*}{$\begin{array}{l}\text { Entrada: } m_{0}, m_{1} \in \mathcal{M} \\
\text { Saída: None } \\
r_{A} \leftarrow \mathbb{Z} / \ell_{A}^{e_{A}} \mathbb{Z} \\
\phi_{A}: E_{0} \rightarrow E_{A} \\
E_{A} \leftarrow E_{0} /\left\langle P_{A}+r_{A} Q_{A}\right\rangle \\
G_{A} \leftarrow \phi_{A}\left(P_{B}\right) ; H_{A} \leftarrow \phi_{A}\left(Q_{B}\right) \\
\mathrm{pk}_{A} \leftarrow\left(E_{A}, G_{A}, H_{A}\right)\end{array}$} & & Entrada: $\sigma \in\{0,1\}$ \\
\hline & & Saída: $m_{\sigma}$ \\
\hline & & $r_{B} \leftarrow \mathbb{Z} / \ell_{B}^{e_{B}} \mathbb{Z}$ \\
\hline & & $\phi_{B}: E_{0} \rightarrow E_{B}$ \\
\hline & & $E_{B} \leftarrow E_{0} /\left\langle P_{B}+r_{B} Q_{B}\right\rangle$ \\
\hline & & $G_{B} \leftarrow \phi_{B}\left(P_{A}\right) ; H_{B} \leftarrow \phi_{B}\left(Q_{A}\right)$ \\
\hline & & $\mathrm{pk}_{B} \leftarrow\left(E_{B}, G_{B}, H_{B}\right)$ \\
\hline & $\mathrm{pk}_{A}$ & Se $G_{A}, H_{A} \notin E_{A}\left[\ell_{B}^{e_{B}}\right]$ \\
\hline & & então rejeite $(\perp)$ \\
\hline & & $U, V \leftarrow E_{B}\left[\ell_{A}^{e_{A}}\right]$ então, \\
\hline & & $\hat{G}_{B} \leftarrow\left(G_{B}-\sigma U\right)$ \\
\hline & & $\hat{H}_{B} \leftarrow\left(H_{B}-\sigma V\right)$ \\
\hline & & $\hat{\mathrm{pk}}_{B} \leftarrow\left(E_{B}, \hat{G}_{B}, \hat{H}_{B}\right)$ \\
\hline \multirow{2}{*}{\multicolumn{3}{|c|}{$\begin{array}{l}\text { Se } \hat{G}_{B}, \hat{H}_{B} \notin E_{B}\left[\ell_{A}^{e_{A}}\right] \\
\quad \text { então, rejeite }(\perp)\end{array}$}} \\
\hline & & \\
\hline \multirow{8}{*}{$\begin{array}{l}\forall i \in\{0,1\} \text { e } U, V \leftarrow{ }_{8} E_{B}\left[\ell_{A}^{e_{A}}\right] \text { então, } \\
\phi_{A_{i}}^{\prime}: E_{B} \rightarrow E_{B A_{i}} \\
E_{B A_{i}} \leftarrow E_{B} /\left\langle\left(\hat{G}_{B}+i U\right)+r_{A}\left(\hat{H}_{B}+i V\right)\right\rangle \\
k_{0}=\mathcal{H}\left(j\left(E_{B A_{0}}\right)\right) \\
k_{1}=\mathcal{H}\left(j\left(E_{B A_{1}}\right)\right)\end{array}$} & & $\phi_{B}^{\prime}: E_{A} \rightarrow E_{A B}$ \\
\hline & & \\
\hline & & $E_{A B} \leftarrow E_{A} /\left\langle G_{A}+r_{B} H_{A}\right\rangle$ \\
\hline & & $k_{\sigma}=\mathcal{H}\left(j\left(E_{A B}\right)\right)$ \\
\hline & & \\
\hline & $c_{0} \leftarrow \mathcal{E}_{k_{0}}\left(m_{0}\right)$ & \\
\hline & $c_{1} \leftarrow \mathcal{E}_{k_{1}}\left(m_{1}\right)$ & \\
\hline & & $m_{\sigma} \leftarrow \mathcal{D}_{k_{\sigma}}\left(c_{\sigma}\right)$ \\
\hline
\end{tabular}

Figura 1. Protocolo $\left(\begin{array}{l}2 \\ 1\end{array}\right)$ - SIOT.

(c) $\phi_{A}\left(P_{B}\right)=G_{A} ; \phi_{A}\left(Q_{B}\right)=H_{A}$.

3. Alice cria um par de chaves $s k_{A}=\left(\phi_{A}, r_{A}\right)$ e $p k_{A}=\left(E_{A}, G_{A}, H_{A}\right)$, isto é, suas chaves privada e pública, respectivamente; e

4. Ela envia para Bob a sua chave pública $p k_{A}=\left(E_{A}, G_{A}, H_{A}\right)$. Ele verifica se $G_{A}, H_{A} \in E_{A}\left[\ell_{B}^{e_{B}}\right]$, isto é, $\ell_{B}^{e_{B}} G_{A}=\ell_{B}^{e_{B}} H_{A}=\mathcal{O}_{A} \in E_{A}$, visto que $\left\{P_{B}, Q_{B}\right\} \subset$ $E_{0}\left[\ell_{B}^{e_{B}}\right]$. Se essa verificação for válida então, Bob aceitará a chave pública de Alice. Caso contrário, a referida chave pública será rejeitada $(\perp)$.

Denota-se $E_{A}=E_{0} /\left\langle P_{A}+r_{A} Q_{A}\right\rangle$ onde $\left|\operatorname{ker}\left(\phi_{A}\right)\right|=\left|\left\langle P_{A}+r_{A} Q_{A}\right\rangle\right|=\ell_{A}^{e_{A}}$, isto é, uma isogenia separável $]^{3}$ de grau $\ell_{A}^{e_{A}}$.

\footnotetext{
${ }^{3}$ Veja [Washington. 2008], Proposição 12.8.
} 


\subsubsection{Setup - Destinatário}

1. Bob escolhe secretamente um valor $r_{B} \in Z / \ell_{B}^{e_{B}} Z$;

2. Ele calcula:

(a) $\operatorname{ker}\left(\phi_{B}\right)=\left\langle P_{B}+r_{B} Q_{B}\right\rangle$;

(b) $\phi_{B}: E_{0} \rightarrow E_{B}$;

(c) $\phi_{B}\left(P_{A}\right)=G_{B} ; \phi_{B}\left(Q_{A}\right)=H_{B}$;

(d) Para um secreto e escolhido $\sigma \in\{0,1\}$, tem-se que $\hat{G}_{B}=\left(G_{B}-\sigma U\right)$ e $\hat{H}_{B}=\left(H_{B}-\sigma V\right)$.

3. Bob cria um par de chaves $s k_{B}=\left(\phi_{B}, r_{B}\right)$ e $\hat{p k_{B}}=\left(E_{B}, \hat{G}_{B}, \hat{H}_{B}\right)$, isto é, suas chaves privada e pública, respectivamente; e

4. Ele envia para Alice a sua chave pública $\hat{p k}_{B}$. Ela executa duas verificações:

- Se $\hat{G}_{B}, \hat{H}_{B} \in E_{B}\left[\ell_{A}^{e_{A}}\right]$, isto é, $\ell_{A}^{e_{A}} \hat{G}_{B}=\ell_{A}^{e_{A}} \hat{H}_{B}=\mathcal{O}_{B} \in E_{B}$, visto que $\left\{P_{A}, Q_{A}\right\} \subset E_{0}\left[\ell_{A}^{e_{A}}\right] \mathrm{e}$

- Se os pontos $U, V \in E_{B}\left[\ell_{A}^{e_{A}}\right]$. Isso assegura que o par de pontos $\left(\hat{G}_{B}+\right.$ $\left.U, \hat{H}_{B}+V\right)$ e $\left(\hat{G}_{B}-U, \hat{H}_{B}-V\right)$ foi gerado por $E_{B}\left[\ell_{A}^{e_{A}}\right]$. Caso contrário, a referida chave pública será rejeitada $(\perp)$ e todo o protocolo é reiniciado para executar uma outra cadeia $w$ de bits.

\subsubsection{Geração de chaves secretas}

\subsubsection{Setup - Alice}

- Alice calcula:

1. Para todo $i \in\{0,1\}, \operatorname{ker}\left(\phi_{A_{i}}^{\prime}\right)=\left\langle\left(\hat{G}_{B}+i U\right)+r_{A}\left(\hat{H}_{B}+i V\right)\right\rangle$;

2. $\phi_{A_{i}}^{\prime}: E_{B} \rightarrow E_{B A_{i}}$;

3. $\jmath_{i} \leftarrow$ invariante $\jmath\left(E_{B A_{i}}\right)$;

4. $k_{i}=\mathcal{H}\left(J_{i}\right)$.

\subsubsection{Setup - Bob}

- Bob calcula:

1. $\operatorname{ker}\left(\phi_{B}^{\prime}\right)=\left\langle G_{A}+r_{B} H_{A}\right\rangle$;

2. $\phi_{B}^{\prime}: E_{B} \rightarrow E_{A B}$;

3. $\jmath_{\sigma} \leftarrow$ invariante $\jmath\left(E_{A B}\right)$

4. $k_{\sigma}=\mathcal{H}\left(\jmath_{\sigma}\right)$.

\subsubsection{Encriptação e Decriptação}

1. Para todo $i \in\{0,1\}$, Alice encripta $m_{i}$. Assim, $c_{i} \leftarrow \mathcal{E}\left(k_{i}, m_{i}\right)$. Após isso, ela envia $\left(c_{0}, c_{1}\right)$ para Bob e;

2. Ele decripta e obtém um único $m_{\sigma}=\mathcal{D}\left(k_{\sigma}, c_{\sigma}\right)$. 


\subsection{Análise de segurança do $\left(\begin{array}{l}2 \\ 1\end{array}\right)$-SIOT}

\subsubsection{Conceitos preliminares para a segurança}

Definição 1. Uma função $\epsilon: \mathbb{N} \rightarrow \mathbb{R}$ é definida como desprezável se para cada polinômio positivo $p(\cdot)$, existe um $N$ tal que para todo $n>N$. Então, $\epsilon(n)<\frac{1}{p(n)}$.

Definição 2. Um conjunto de probabilidades $\mathcal{X}=\{\mathcal{X}(n, a)\}$ é uma sequência infinita de variáveis aleatórias indexadas por $n \in \mathbb{N}$ e a $\in\{0,1\}^{*}$. O valor $\underline{n}$ representa um parâmetro de segurança e a representa as entradas das partes ou participantes.

Definição 3. Dois conjuntos de distribuição $\mathcal{X}=\{\mathcal{X}(n, a)\}$ e $\mathcal{Y}=\{\mathcal{Y}(n, a)\}$ são computacionalmente indistinguíveis, denotado por $\mathcal{X} \stackrel{\mathrm{c}}{\equiv} \mathcal{Y}$, se para cada algoritmo de tempo não-polinomial $\mathcal{D}$, existe uma função desprezável $\epsilon(\cdot)$ tal que para cada $n \in \mathbb{N} e$ $a \in\{0,1\}^{*}$. Então, $|\operatorname{Pr}[\mathcal{D}(\mathcal{X}(n, a))=1]-\operatorname{Pr}[\mathcal{D}(\mathcal{Y}(n, a))=1]| \leqslant \epsilon(n)$.

\subsubsection{Problemas computacionais de isogenias entre curvas elípticas supersingulares}

Nesta seção, serão apresentados os problemas de decisão e computacionais que garantem a segurança do protocolo SIDH. Para maiores detalhes, o leitor deve consul$\operatorname{tar}$ [Feo et al. 2014]. Como será visto a seguir, a segurança do $\left(\begin{array}{l}2 \\ 1\end{array}\right)$-SIOT está fundamentada nesses problemas.

Problema 1 (Problema Decisional de Isogenia Supersingular - DSSI). Seja $E_{A}$ uma outra curva supersingular definida sobre $\mathbb{F}_{p^{2}}$. Decida se $E_{A}$ e $E_{0}$ são isogeneas de grau $\ell_{A}^{e_{A}}$.

Problema 2 (Problema Computational de Isogenia Supersingular - CSSI). Seja $\phi_{A}: E_{0} \rightarrow E_{A}$ uma isogenia cujo kernel $]^{4}$ é $R_{A}=\left\langle\left[m_{A}\right] P_{A}+\left[r_{A}\right] Q_{A}\right\rangle$ onde $m_{A}$ e $r_{A}$ são escolhidos aleatoriamente de $\mathbb{Z} / \ell_{A}^{e_{A}} \mathbb{Z}$ e ambos não são divisíveis por $\ell_{A}^{e_{A}}$. Seja ainda a chave pública $\left(E_{A}, G_{A}, H_{A}\right)$. Determine $R_{A}$.

Problema 3 (Problema Computacional Diffie-Helmann Supersingular - SSCDH). Sejam $\phi_{A}: E_{0} \rightarrow E_{A}$ uma isogenia cujo kernel é $R_{A}=\left\langle P_{A}+\left[r_{A}\right] Q_{A}\right\rangle$, para algum $r_{A} \in \mathbb{Z} / \ell_{A}^{e_{A}} \mathbb{Z}$ e $\phi_{B}: E_{0} \rightarrow E_{B}$ uma isogenia cujo kernel é $R_{B}=\left\langle P_{B}+\left[r_{B}\right] Q_{B}\right\rangle$, para algum $r_{B} \in \mathbb{Z} / \ell_{B}^{e_{B}} \mathbb{Z}$. Dado as chaves públicas $\left(E_{A}, G_{A}, H_{A}\right)$ e $\left(E_{B}, G_{B}, H_{B}\right)$. Determine o invariante j de $E_{0} /\left\langle P_{A}+\left[r_{A}\right] Q_{A}, P_{B}+\left[r_{B}\right] Q_{B}\right\rangle$.

Problema 4 (Problema Decisional Diffie-Hellman de Isogenia Supersingular - SSDDH). Dado uma amostra com probabilidade 1/2 de uma das duas seguintes distribuições:

1. $\left(E_{A}, E_{B}, G_{A}, H_{A}, G_{B}, H_{B}, E_{A B}\right)$ onde $E_{A}, E_{B}, G_{A}, H_{A}, G_{B}$ e $H_{B}$ como no problema 3 e,

$$
E_{A B} \simeq E_{0} /\left\langle P_{A}+\left[r_{A}\right] Q_{A}, P_{B}+\left[r_{B}\right] Q_{B}\right\rangle,
$$

2. $\left(E_{A}, E_{B}, G_{A}, H_{A}, G_{B}, H_{B}, E_{C}\right)$ onde $E_{A}, E_{B}, G_{A}, H_{A}, G_{B}$ e $H_{B}$ como no problema $3 e$,

\footnotetext{
${ }^{4}$ No protocolo $\left(\begin{array}{l}2 \\ 1\end{array}\right)$-SIOT, utilizou-se o kernel $\left\langle P_{A}+\left[r_{A}\right] Q_{A}\right\rangle$.
} 


$$
E_{C} \simeq E_{0} /\left\langle P_{A}+\left[\hat{r}_{A}\right] Q_{A}, P_{B}+\left[\hat{r}_{B}\right] Q_{B}\right\rangle,
$$

onde $r_{A}, \hat{r}_{A} \in \mathbb{Z} / \ell_{A}^{e_{A}} \mathbb{Z}$ e $r_{B}, \hat{r}_{B} \in \mathbb{Z} / \ell_{B}^{e_{B}} \mathbb{Z}$ e $r_{A} \neq \hat{r}_{A}$ e $r_{B} \neq \hat{r}_{B}$. Além disso, ambos $\left(r_{A}, \hat{r}_{A}\right)$ e $\left(r_{B}, \hat{r}_{B}\right)$ não são divisíveis por $\ell_{A}^{e_{A}} \quad e \quad \ell_{B}^{e_{B}}$, respectivamente. Determine a qual distribuição a tupla pertence.

Nota: Cabe ressaltar que como cada amostra tem uma probabilidade de 1/2 então, pela definição 3 tem-se que:

$$
\left\{E_{A}, E_{B}, G_{A}, H_{A}, G_{B}, H_{B}, E_{A B}\right\} \stackrel{\mathbf{c}}{\equiv}\left\{E_{A}, E_{B}, G_{A}, H_{A}, G_{B}, H_{B}, E_{C}\right\} .
$$

\subsubsection{Notações para a análise de segurança}

$\mathrm{Na}$ análise de segurança do protocolo proposto, as seguintes notações serão utilizadas, a saber:

1. Aplicação da fórmula de Vélu ${ }^{5} \rightarrow$ Vélu's formula $\{k e r(\phi), E\}$;

2. De acordo com [Kalai 2005], em um protocolo OT, a substituição de uma das mensagens do par de entrada $m_{0}$ e $m_{1}$ por outra mensagem $m$ deve passar despercebida pelo destinatário. Dado $\tau \in \mathcal{M}$ e $\sigma \in\{0,1\}$, a compreensão ou visão de Alice na execução de um protocolo OT é denotada por $\left\{\Omega_{\text {Alice }}\left(\operatorname{Alice}\left(1^{n}, \tau\right), \operatorname{Bob}\left(1^{n}, \sigma\right)\right)\right\}$, onde um parâmetro de segurança é definido por $1^{n}$. Similarmente, denotamos a compreensão ou visão de Bob por $\left\{\Omega_{B o b}\left(\operatorname{Alice}\left(1^{n}, \tau\right), \operatorname{Bob}\left(1^{n}, \sigma\right)\right)\right\}$ e;

3. Ambos Alice e Bob como usuários desonestos são denotados por Alice* e Bob*, respectivamente.

\subsubsection{Alguns requisitos para a Análise de Segurança}

Em princípio, qualquer protocolo seguro deverá resistir a qualquer ataque malicioso. Assim, para comprovar que um protocolo OT seja seguro, [Hazay and Lindell. 2010] afirmam que os mais importantes requisitos em qualquer protocolo de segurança são a corretude e privacidade.

Corretude. No $\left(\begin{array}{l}2 \\ 1\end{array}\right)$-SIOT, suponhamos que tanto Alice quanto Bob são partes honestas na execução do protocolo. Sejam $\sigma, i \in\{0,1\}$ tal que $\sigma=i$. Então, a corretude do protocolo segue pelas identidades abaixo.

$$
\begin{aligned}
\jmath\left(E_{B A_{i}}\right) & \simeq \jmath\left(E_{B} /\left\langle\left(G_{B}-\sigma \cdot U+i \cdot U\right)+r_{A} \cdot\left(H_{B}-\sigma \cdot V+i \cdot V\right)\right\rangle\right) \\
& \simeq \jmath\left(\phi_{B}\left(E_{0}\right) /\left\langle\left(\phi_{B}\left(P_{A}\right)-\sigma \cdot U+i \cdot U\right)+r_{A}\left(\phi_{B}\left(Q_{A}\right)-\sigma \cdot V+i \cdot V\right)\right\rangle\right) \\
& \simeq \jmath\left(\phi_{B}\left(E_{0}\right) /\left\langle\phi_{B}\left(P_{A}\right)+r_{A} \cdot \phi_{B}\left(Q_{A}\right)\right\rangle\right) \\
& \simeq \jmath\left(\phi_{B}\left(E_{0}\right) /\left\langle\phi_{B}\left(P_{A}+r_{A} \cdot Q_{A}\right)\right\rangle\right) \\
& \simeq \jmath\left(\phi_{A_{i}}^{\prime}\left(\phi_{B}\left(E_{0}\right)\right)\right) \simeq \jmath\left(\phi_{A}\left(E_{0}\right) /\left\langle\phi_{A}\left(P_{B}+r_{B} \cdot Q_{B}\right)\right\rangle\right) \\
& \simeq \jmath\left(\phi_{A_{i}}^{\prime}\left(\phi_{B}\left(E_{0}\right)\right)\right) \simeq \jmath\left(E_{B} /\left\langle\phi_{A}\left(P_{B}\right)+r_{B} \cdot \phi_{B}\left(Q_{B}\right)\right\rangle\right) \\
& \simeq \jmath\left(\phi_{A_{i}}^{\prime}\left(\phi_{B}\left(E_{0}\right)\right)\right) \simeq \jmath\left(E_{B} /\left\langle G_{A}+r_{B} \cdot H_{A}\right\rangle\right) \simeq \jmath\left(\phi_{B}^{\prime}\left(\phi_{A}\left(E_{0}\right)\right)\right) \simeq \jmath\left(E_{A B}\right) .
\end{aligned}
$$

Privacidade. No protocolo $\left(\begin{array}{l}2 \\ 1\end{array}\right)$-SIOT, as escolhas de Bob não devem ser conhecidas por Alice. Além disso, no final da execução do referido protocolo, Bob não poderá obter

\footnotetext{
${ }^{5}$ Veja [Galbraith. 2012], corolário 25.1.7.
} 
nenhum conhecimento sobre a mensagem que ele não decriptou. Cabe ressaltar que essa privacidade decorre da dificuldade de resolução dos problemas computacionais vistos na seção 2.5.2. Por fim, para complementar a prova de segurança do protocolo $\left(\begin{array}{l}2 \\ 1\end{array}\right)$-SIOT , elaborou-se o Teorema 1 a seguir:

Teorema 1. Suponha que os problemas computacionais de isogenia supersingular CSSI, $S S C D H$ e SSDDH são difíceis no grupo $E\left(\mathbb{F}_{p^{2}}\right)$. Então, o protocolo $\left(\begin{array}{l}2 \\ 1\end{array}\right)$-SIOT assegura a privacidade entre duas partes.

$\begin{array}{llllll}\text { Demonstração. A prova consiste em adaptar a definição } & 2.6 .1\end{array}$ de [Hazay and Lindell. 2010] ao protocolo $\left(\begin{array}{l}2 \\ 1\end{array}\right)$-SIOT pela compatibilização com os problemas computacionais citados acima. Sejam duas mensagens, $m_{0}$ e $m_{1}$, entre duas partes (Alice e Bob). Um protocolo OT é privado se os seguintes requisitos forem válidos:

\section{i. Não-trivialidade}

Se Alice e Bob seguem corretamente o protocolo, então depois de uma execução em que Alice possui como entrada os textos claros $m_{0}, m_{1} \in \mathcal{M}$ e Bob possui como entrada um bit $\sigma \in\{0,1\}$, a saída de Bob será um único texto decriptado $m_{\sigma}$. Em outras palavras, Bob recebe a chave pública $p k_{A}$ e o par de textos encriptados $\left(c_{0}, c_{1}\right)$ de Alice. Relembrando que $p k_{A} \leftarrow\left(E_{A}, G_{A}, H_{A}\right)$ e $c_{\sigma} \leftarrow \mathcal{E}\left(k_{\sigma}, m_{\sigma}\right)$ são bem definidos no protocolo $\left(\begin{array}{l}2 \\ 1\end{array}\right)$-SIOT . Assim, a não-trivialidade provém da seguinte relação:

$$
\begin{gathered}
\text { Vélu's formula }\left\{\left\langle G_{A}+r_{B} H_{A}\right\rangle, E_{A}\right\} \Rightarrow E_{A B} \therefore \\
\mathcal{H}\left(\jmath\left(E_{A B}\right)\right) \Rightarrow k_{\sigma} .
\end{gathered}
$$

Portanto, Bob recupera $k_{\sigma}$ o que implica em $m_{\sigma} \leftarrow \mathcal{D}\left(k_{\sigma}, c_{\sigma}\right)$ tal que $\sigma$ é um único valor escolhido secretamente por ele. Além disso, após receber $p k_{A}$, Bob não será capaz de calcular a chave privada $\left(\phi_{A}, r_{A}\right)$ de Alice. Caso contrário, haveria uma violação da hipótese de dificuldade do problema CSSI.

\section{ii. Privacidade no caso de Bob ser uma parte desonesta}

Seja $\hat{p k}_{B} \leftarrow\left(E_{B}, \hat{G}_{B}, \hat{H}_{B}\right)$ a chave pública de Bob enviada para Alice. Relembre que $\hat{G}_{B} \leftarrow G_{B}, \hat{H}_{B} \leftarrow H_{B}$, se $\sigma=0$ e $\hat{G}_{B} \leftarrow\left(G_{B}-U\right), \hat{H}_{B} \leftarrow\left(H_{B}-\right.$ $V)$, se $\sigma=1$. Além disso, há a chave pública $p k_{A} \leftarrow\left(E_{A}, G_{A}, H_{A}\right)$ de Alice enviada para Bob e um único valor do invariante $j_{\sigma} \leftarrow j$ (Vélu's formula $\left\{\left\langle G_{A}+\right.\right.$ $\left.\left.r_{B} H_{A}\right\rangle, E_{A}\right\}$ ) calculado por Bob ao receber a bem definida chave pública $p k_{A}$ de Alice. Após isso, $\forall i \in\{0,1\}$, Alice computará $\jmath_{i} \leftarrow \jmath\left(V\right.$ élu's formula $\left\{\left\langle\left(\hat{G}_{B}+\right.\right.\right.$ $\left.\left.\left.i U)+r_{A}\left(\hat{H}_{B}+i V\right)\right\rangle, E_{B}\right\}\right)$, isto é, $\jmath_{0}$ e $\jmath_{1}$.

Adicionalmente, Alice compartilhará uma única chave secreta com Bob. Assim, a privacidade de Alice é baseada no seguinte fato: Bob não pode computar ambos os valores dos invariantes $\jmath_{0}$ and $\jmath_{1}\left(\jmath_{0} \neq \jmath_{1}\right)$ se a hipótese do problema SSCDH é difícil. Em outras palavras, Bob será capaz de computar um único valor de um invariante $\jmath_{\sigma}$.

Além disso, seja $\sigma \in\{0,1\}$ uma entrada auxiliar e uma outra entrada com a tupla $m_{0}, m_{1}, m \in \mathcal{M}$. Assim, uma outra forma de visualizar a privacidade de Alice é que a primeira mensagem de Bob, denotada por Bob* $\left(1^{n}, \sigma\right)$, determina qual dos textos $m_{0}$ ou $m_{1}$ será recebido por ele. Por exemplo, se é determinado que o texto $m_{0}$ deve ser recebido, então a visualização de Bob quando a entrada de Alice 
corresponde a $\left(m_{0}, m_{1}\right)$ é indistinguível de sua visualização quando a entrada de Alice corresponde a $\left(m_{0}, m\right)$. Evidentemente, isso implica que Bob não pode aprender nada sobre o texto $m_{1}$ quando o texto $m_{0}$ é recebido e vice-versa. Então,

$\left.\left\{\Omega_{\text {Bob* }^{*}} \operatorname{Alice}\left(1^{n},\left(m_{0}, m_{1}\right)\right) ; B o b^{*}\left(1^{n}, \sigma\right)\right)\right\}_{n \in \mathbb{N}} \stackrel{\text { c }}{=}\left\{\Omega_{\text {Bob }^{*}}\left(\operatorname{Alice}\left(1^{n},\left(m_{0}, m\right)\right) ; B o b^{*}\left(1^{n}, \sigma\right)\right)\right\}_{n \in \mathbb{N}}$

$\left.\left\{\Omega_{B o b^{*}}\left(\operatorname{Alice}\left(1^{n},\left(m_{0}, m_{1}\right)\right) ; B o b^{*}\left(1^{n}, \sigma\right)\right)\right\}_{n \in \mathbb{N}} \stackrel{\text { ou }}{\equiv}\left\{\Omega_{B o b^{*}} \operatorname{Alice}\left(1^{n},\left(m, m_{1}\right)\right) ; B o b^{*}\left(1^{n}, \sigma\right)\right)\right\}_{n \in \mathbb{N}}$

\section{iii. Privacidade no caso de Alice ser uma parte desonesta}

Observe que este requisito mostra que Alice não consegue distinguir as possíveis escolhas secretas de Bob, isto é, quando o bit $\sigma$ assume o valor 0 ou 1, isso é indistinguível na visão de Alice. Em outras palavras, ela visualiza simplesmente uma chave pública $\hat{p k_{B}}$. Assim, a privacidade do destinatário será verificada pelo seguinte Lema:

Lema 1. Ao receber a chave pública $\hat{p k}_{B}$ de Bob, Alice não pode adivinhar o valor do bit $\sigma$ a uma probabilidade maior que $1 / 2+\epsilon(n)$, para alguma função desprezável $\epsilon(n) e \forall n \in \mathbb{N}$.

Demonstração. Suponha que no recebimento da chave pública $\hat{p k}_{B}$ e não conhecendo o valor secreto do bit $\sigma$ de Bob, Alice não pode distinguir entre os pares de tupla $\left\{\left(E_{B}, G_{B}, H_{B}\right)\right\}$ e $\left\{\left(E_{B},\left(G_{B}-U\right),\left(H_{B}-V\right)\right\}\right.$, isto é, para algum $\hat{G}_{B}, \hat{H}_{B} \in E_{B}\left[\ell_{A}^{e_{A}}\right]$ tal que $\operatorname{Pr}\left[\left(E_{B}, G_{B}, H_{B}\right)=\left(E_{B}, \hat{G}_{B}, \hat{H}_{B}\right)\right]=\operatorname{Pr}\left[\left(E_{B}, G_{B}-\right.\right.$ $\left.\left.\left.U, H_{B}-V\right)=\left(E_{B}, \hat{G}_{B}, \hat{H}_{B}\right)\right)\right]$ é independente do valor de $\sigma$. Assim, a dificuldade dessa indistinguibilidade entre essas tuplas é baseada na hipótese de dificuldade do problema SSDDH. Então, tem-se que:

$$
\left\{\left(E_{B}, G_{B}, H_{B}\right)\right\} \stackrel{\text { c }}{\equiv}\left\{E_{B},\left(G_{B}-U\right),\left(H_{B}-V\right)\right\}
$$

De acordo com [Rogaway 2004], um diferenciador é um algoritmo probabilístico que descreve a vantagem de um adversário. Assim, suponha que, por contradição, existe um diferenciador probabilístico $\Theta$ de tempo polinomial e uma função nãodesprezável $\epsilon$ tal que para todo $n$, tem-se que

$$
\left|P_{r}\left[\Theta\left(E_{B}, G_{B}, H_{B}\right)=1\right]-P_{r}\left[\Theta\left(E_{B},\left(G_{B}-U\right),\left(H_{B}-V\right)\right)=1\right]\right| \geqslant \epsilon(n),
$$

Desta forma, subtraindo e somando o seguinte termo,

$$
P_{r}\left[\Theta\left(E_{B},\left(G_{B}-U-R\right),\left(H_{B}-V-S\right)\right)=1\right]
$$

Tem-se que

$\left|P_{r}\left[\Theta\left(E_{B}, G_{B}, H_{B}\right)=1\right]-P_{r}\left[\Theta\left(E_{B},\left(G_{B}-U\right),\left(H_{B}-V\right)\right)=1\right]\right| \leqslant$ $\left|P_{r}\left[\Theta\left(E_{B}, G_{B}, H_{B}\right)=1\right]-P_{r}\left[\Theta\left(E_{B},\left(G_{B}-U-R\right),\left(H_{B}-V-S\right)\right)=1\right]\right|+$ $\mid P_{r}\left[\Theta\left(E_{B},\left(G_{B}-U-R\right),\left(H_{B}-V-S\right)\right)=1\right]-P_{r}\left[\Theta\left(E_{B},\left(G_{B}-U\right),\left(H_{B}-V\right)\right)=\right.$ $1] \mid$

Por contradição, suponhamos que

$$
\left|P_{r}\left[\Theta\left(E_{B}, G_{B}, H_{B}\right)=1\right]-P_{r}\left[\Theta\left(E_{B},\left(G_{B}-U-R\right),\left(H_{B}-V-S\right)\right)=1\right]\right| \geqslant \frac{\epsilon(n)}{2}
$$

${ }^{6} R, S \in E_{B}\left[\ell_{A}^{e_{A}}\right]$. 


$$
\left|P_{r}\left[\Theta\left(E_{B},\left(G_{B}-U-R\right),\left(H_{B}-V-S\right)\right)=1\right]-P_{r}\left[\Theta\left(E_{B},\left(G_{B}-U\right),\left(H_{B}-V\right)\right)=1\right]\right| \geqslant \frac{\epsilon(n)}{2}
$$

Suponha que a equação 1 seja válida. Então, pode-se construir um diferenciador $\tilde{\Theta}$ para o problema SSDDH que funciona da seguinte forma: Após receber a chave pública $\hat{p} k_{B} \leftarrow\left(E_{B},\left(G_{B}-U\right),\left(H_{B}-V\right)\right)$, o diferenciador $\tilde{\Theta}$ aleatoriamente escolhe os pares de pontos $R, S$ e, assim, $\hat{p} k_{B}^{\prime} \leftarrow\left(E_{B},\left(G_{B}-U-R\right),\left(H_{B}-V-S\right)\right)$. Por outro lado, se $\hat{p} k_{B} \leftarrow\left(E_{B}, G_{B}, H_{B}\right)$ então, $\hat{p} k_{B}^{\prime} \leftarrow\left(E_{B},\left(G_{B}-R\right),\left(H_{B}-S\right)\right)$. Observe que os pares de pontos $U$ e $V$ não são utilizados nesta última tupla. Contudo, esses pontos, assim como os outros pontos $R$ e $S$, são oriundos do mesmo grupo $E_{B}\left[\ell_{A}^{e_{A}}\right]$ e poderiam ser também aleatoriamente escolhidos pelo diferenciador $\tilde{\Theta}$, digo os pontos $U$ e $V$. Portanto, tem-se que $\hat{p} k_{B}^{\prime} \leftarrow\left(E_{B}, G_{B}, H_{B}\right)$ e,

$$
\begin{gathered}
\left|P_{r}\left[\tilde{\Theta}\left(E_{B}, G_{B}, H_{B}\right)=1\right]-P_{r}\left[\tilde{\Theta}\left(E_{B},\left(G_{B}-U\right),\left(H_{B}-V\right)\right)=1\right]\right|= \\
\left|P_{r}\left[\Theta\left(E_{B}, G_{B}, H_{B}\right)=1\right]-P_{r}\left[\Theta\left(E_{B},\left(G_{B}-U-R\right),\left(H_{B}-V-S\right)\right)=1\right]\right| \geqslant \frac{\epsilon(n)}{2}
\end{gathered}
$$

o que contradiz com as hipóteses de dificuldade do problema SSDDH. De modo análogo, tem-se um resultado similar caso a equação 2 como válida.

A prova da privacidade de Bob é concluída por observar que $\left\{\left(E_{B}, G_{B}, H_{B}\right)\right\}$, $\left\{\left(E_{B},\left(G_{B}-U\right),\left(H_{B}-V\right)\right)\right\}$, independentemente do valor de $\sigma$, são indistinguíveis na visualização de Alice. Em outras palavras, seja $\tau \in\{0,1\}^{*}$ uma entrada auxiliar então,

$$
\left\{\Omega_{\text {Alice* }}\left(\operatorname{Alice}^{*}\left(1^{n}, \tau\right), \operatorname{Bob}\left(1^{n}, 0\right)\right)\right\} \stackrel{\mathbf{c}}{\equiv}\left\{\Omega_{\text {Alice* }}\left(\operatorname{Alice}^{*}\left(1^{n}, \tau\right), \operatorname{Bob}\left(1^{n}, 1\right)\right)\right\} .
$$

De acordo com o Lema 1, tem-se que a privacidade de Bob fundamenta-se na hipótese de dificuldade do problema SSDDH sobre um grupo $E_{B}\left[\ell_{A}^{e_{A}}\right]$.

\subsubsection{Análise algébrica de segurança do protocolo $\left(\begin{array}{l}2 \\ 1\end{array}\right)$-SIOT}

Considere o hipotético caso de uma Alice desonesta onde ela tentará utilizar um diferenciador baseado em emparelhamento de Weil para descobrir o valor secreto do bit $\sigma$ de um Bob honesto. Em uma segunda situação hipotética, as funções são invertidas, isto é, Alice será considerada como uma remetente honesta e Bob como um destinatário desonesto. Desta forma, uma análise algébrica é desenvolvida de tal forma que certas condições devem ser obedecidas para que Bob não seja capaz de decriptar ambas as mensagens transmitidas por Alice. 


\subsubsection{Prevenindo contra uma Alice desonesta que utiliza um diferenciador base- ado em emparelhamento de Weil}

Considere a situação em que Alice, atuando como um remetente desonesto, recebe a chave pública $\left(E_{B}, \hat{G}_{B}, \hat{H}_{B}\right)$ de Bob. Assim, em princípio, ela não tem o conhecimento se recebeu a chave pública $\left(E_{B}, G_{B}, H_{B}\right)$ ou $\left(E_{B}, G_{B}-U, H_{B}-V\right)$. Desta forma, Alice utilizará o emparelhamento de Weil para diferenciar esses dois valores. No que se segue, todos os emparelhamentos tem ordem $\ell_{A}^{e_{A}}$. Durante a execução do protocolo, os pontos $G_{B}=\phi_{B}\left(P_{A}\right)$ e $H_{B}=\phi_{B}\left(Q_{A}\right)$ são bem definidos para satisfazer a relação $e\left(G_{B}, H_{B}\right)=$ $e\left(P_{A}, Q_{A}\right)^{\ell_{A}^{e_{A}}}$. Se essa relação não for válida para ambos $\left(\hat{G}_{B}, \hat{H}_{B}\right)$ ou $\left(\hat{G}_{B}+U, \hat{H}_{B}+\right.$ $V)$, isso revelará qual a chave pública que foi escolhida por Bob. Em geral, Alice pode somar qualquer múltiplo de $(U, V)$ em $\left(\hat{G}_{B}, \hat{H}_{B}\right)$ e observar por uma incompatibilidade. Assim, é preciso ter $e\left(\hat{G}_{B}+\lambda U, \hat{H}_{B}+\lambda V\right)=e\left(G_{B}, H_{B}\right)=e\left(P_{A}, Q_{A}\right)^{\ell_{A}^{e}}$ para algum $\lambda \in \mathbb{Z} / \ell_{A}^{e_{A}} \mathbb{Z}$. Relembrando que $U, V, G_{B}, H_{B} \in E_{B}\left[\ell_{A}^{e_{A}}\right]$, então eles podem ser escritos como uma combinação linear, isto é, $U=\alpha G_{B}+\beta H_{B}$ e $V=\gamma G_{B}+\delta H_{B}$ tal que $\alpha, \beta, \gamma, \delta \in \mathbb{Z} / \ell_{A}^{e_{A}} \mathbb{Z}$. Assim, isto significa que:

$$
\begin{aligned}
e\left(G_{B}+\lambda U, H_{B}+\lambda V\right) & =e\left(G_{B}+\lambda \alpha G_{B}+\lambda \beta H_{B}, H_{B}+\lambda \gamma G_{B}+\lambda \delta H_{B}\right) \\
& =e\left((1+\lambda \alpha) G_{B}+\lambda \beta H_{B}, \lambda \gamma G_{B}+(1+\lambda \delta) H_{B}\right) \\
& =e\left((1+\lambda \alpha) G_{B}, \lambda \gamma G_{B}\right) \\
& \cdot e\left((1+\lambda \alpha) G_{B},(1+\lambda \delta) H_{B}\right) \\
& \cdot e\left(\lambda \beta H_{B}, \lambda \gamma G_{B}\right) \\
& \cdot e\left(\lambda \beta H_{B},(1+\lambda \delta) H_{B}\right) \\
= & 1 \\
& \cdot e\left(G_{B}, H_{B}\right)^{(1+\lambda \alpha)(1+\lambda \delta)} \\
& \cdot e\left(H_{B}, G_{B}\right)^{\lambda \beta \lambda \gamma} \\
& \cdot 1 \\
= & e\left(G_{B}, H_{B}\right)^{(1+\lambda \alpha)(1+\lambda \delta)-\lambda^{2} \beta \gamma} \\
= & e\left(G_{B}, H_{B}\right)
\end{aligned}
$$

Desta forma, é necessário que $(1+\lambda \alpha)(1+\lambda \delta)-\lambda^{2} \beta \gamma=1\left(\bmod \ell_{A}^{e_{A}}\right)$, ou equivalentemente $\lambda(\alpha+\delta)+\lambda^{2}(\alpha \delta-\beta \gamma)=0\left(\bmod \ell_{A}^{e_{A}}\right)$. Isto deve ser válido para qualquer $\lambda$, em particular para aqueles que são invertíveis $\bmod \ell_{A}^{e_{A}}$, e então, deve ser válido que $\lambda(\alpha \delta-\beta \gamma)=-(\alpha+\delta)\left(\bmod \ell_{A}^{e_{A}}\right)$. Mais uma vez, isto pode somente ser válido para al$\operatorname{gum} \lambda$ se $\alpha \delta-\beta \gamma=0\left(\bmod \ell_{A}^{e_{A}}\right)$ e $\alpha+\delta=0\left(\bmod \ell_{A}^{e_{A}}\right)$, ou equivalentemente, $\delta=-\alpha$ $\left(\bmod \ell_{A}^{e_{A}}\right)$ e $\alpha^{2}+\beta \gamma=0\left(\bmod \ell_{A}^{e_{A}}\right)$. Portanto, tais condições devem ser obedecidas para evitar que Alice descubra o valor secreto do bit $\sigma$ de Bob.

\subsubsection{Prevenindo contra uma possível decriptação indesejada de um Bob deso- nesto}

Relembre que $U, V \in E_{B}\left[\ell_{A}^{e_{A}}\right]$ são pontos linearmente independentes e que tais pontos podem ser expressos por $U=\alpha G_{B}+\beta H_{B}, V=\gamma G_{B}+\delta H_{B}$. Suponha que 
Alice receba a chave pública $\left(E_{B}, \hat{G}_{B}, \hat{H}_{B}\right)$ de Bob. Assim, Alice irá computar a isogenia $\phi_{A_{0}}^{\prime}: E_{B} \rightarrow E_{B A_{0}}$, com grau $\ell_{A}^{e_{A}}$, cujo kernel é $\operatorname{ker}\left(\phi_{A_{0}}^{\prime}\right)=\left\langle G_{B}+r_{A} H_{B}\right\rangle$. Analogamente, ela irá calcular a isogenia $\phi_{A_{1}}^{\prime}: E_{B} \rightarrow E_{A B_{1}}$, com grau $\ell_{A}^{e_{A}}$, cujo kernel é $\operatorname{ker}\left(\phi_{A_{1}}\right)=\left\langle\left(G_{B}+U\right)+r_{A}\left(H_{B}+V\right)\right\rangle$. Ressalta-se que 7 se $\operatorname{ker}\left(\phi_{A_{0}}^{\prime}\right) \subseteq$ $\operatorname{ker}\left(\phi_{A_{1}}\right)$ então, a isogenia $E_{B A_{0}}$ é isomorfa com relação a isogenia $E_{B A_{1}}$, isto é, denotase $E_{B A_{0}} \cong E_{B A_{1}}$. Adicionalmente, se $\phi_{A_{1}}$ é separável, então existe uma única isogenia $\hat{\phi_{A}}: E_{B A_{0}} . \rightarrow E_{B A_{1}}$. Nesse momento, considere $\left(G_{B}+U\right)+r_{A}\left(H_{B}+V\right)=$ $\left(G_{B}+\alpha G_{B}+\beta H_{B}\right)+r_{A}\left(H_{B}+\gamma G_{B}+\delta H_{B}\right)=\left(1+\alpha+\gamma r_{A}\right) G_{B}+\left(r_{A}+\beta+\delta r_{A}\right) H_{B}$. Assim, por inspeção, esse ponto somente pode pertencer ao kernel $\left\langle G_{B}+r_{A} H_{B}\right\rangle$ considerando as seguintes condições:

1. $\left(1+\alpha+\gamma r_{A}\right)$ é invertível $\bmod \ell_{A}^{e_{A}}$ (i.e. if $\left.\ell_{A} \nmid 1+\alpha+\gamma r_{A}\right)$;

2. $\left(r_{A}+\beta+\delta r_{A}\right) /\left(1+\alpha+\gamma r_{A}\right)=r_{A}\left(\bmod \ell_{A}^{e_{A}}\right)$, que significa $\gamma r_{A}^{2}+(\alpha+\delta) r_{A}-\beta=$ $0\left(\bmod \ell_{A}^{e_{A}}\right) \mathrm{e}, \mathrm{assim}, \gamma r_{A}^{2}+(\alpha-\delta) r_{A}-\beta=0\left(\bmod \ell_{A}\right)$. Então, uma simples restrição nos coeficientes assegura que essa última equação não tenha solução. Desta forma, arbitrariamente define-se $\ell_{A} \mid \gamma$ and $\ell_{A} \mid(\alpha-\delta)$, no entanto $\ell_{A} \nmid \beta$.

Portanto, é importante que a equação $\gamma r_{A}^{2}+(\alpha-\delta) r_{A}-\beta=0\left(\bmod \ell_{A}\right)$ não tenha solução porque, caso contrário, se Alice e Bob não podem controlar os coeficientes $\alpha, \beta, \gamma, \delta$, além de garantir as condições supracitadas, Bob poderia ser capaz de decriptar ambas as mensagens de Alice.

\subsubsection{Sintetizando as condições algébricas}

Nesta seção, são consideradas as três condições, envolvendo os coeficientes $\alpha, \beta, \gamma \mathrm{e}$ $\delta$, baseadas nas equações obtidas nas seções 2.5.5.1 e 2.5.5.2 para garantir a segurança do protocolo $\left(\begin{array}{l}2 \\ 1\end{array}\right)$-SIOT quando Alice e Bob são partes desonestas. Assim, as premissas dos referidos coeficientes são alcançadas de forma a assegurar que Alice não seja capaz de obter a escolha secreta do valor do bit $\sigma$ de Bob e que ele não seja capaz de decriptar ambas as mensagens cifradas do par $c_{0}$ e $c_{1}$ enviadas por Alice. Combinando essas relações, temse $\gamma=-\alpha^{2} / \beta\left(\bmod \ell_{A}^{e_{A}}\right)$ desde que $\beta$ é invertível $\bmod \ell_{A}^{e_{A}}$. Em particular, isso significa que $V=-(\alpha / \beta) U$.

\section{Conclusão da segurança do protocolo $\left(\begin{array}{l}2 \\ 1\end{array}\right)$-SIOT}

A prova de segurança do protocolo $\left(\begin{array}{l}2 \\ 1\end{array}\right)$-SIOT foi fundamentada em 03 (três) partes, a saber:

i. As características de segurança herdadas do protocolo de [Feo et al. 2014], isto é, os problemas computacionais mencionados na seção 2.5.2;

ii. A prova da privacidade entre um remetente e destinatário em um canal de comunicação, por meio do Teorema $1 \mathrm{e}$;

iii. Uma análise algébrica que utilizou o emparelhamento de Weil que definiu algumas condições necessárias para que um remetente desonesto não fraude a segurança do $\left(\begin{array}{l}2 \\ 1\end{array}\right)$-SIOT em relação a um destinatário desonesto e vice-versa.

\footnotetext{
${ }^{7}$ Veja [Galbraith. 2012], Teorema 9.6.18 e [Washington. 2008], Proposição 12.12.
} 


\section{Implementação do protocolo proposto}

O protocolo $\left(\begin{array}{l}2 \\ 1\end{array}\right)$-SIOT foi implementado na linguagem python, utilizando um MacBook Air com um processador de $1.6 \mathrm{GHz}$ Intel Core i.5, memória de $4 \mathrm{~GB}, 1.600 \mathrm{MHz}$ e DDR3. A tabela 1 mostra os valores dos números primos $p$ que foram usados na implementação do protocolo proposto.

Tabela 1. Valores utilizados de $p$ no protocolo $\left(\begin{array}{l}2 \\ 1\end{array}\right)$-SIOT.

\begin{tabular}{ccc}
\hline$p=\ell_{A}^{e_{A}} \ell_{B}^{e_{B}} f \pm 1$ & Valor & Tamanho (bits) \\
\hline$\left(3^{4} \cdot 5^{3} \cdot 4\right)-1$ & 40.499 & 16 \\
$\left(3^{6} \cdot 5^{3} \cdot 4\right)-1$ & 364.499 & 19 \\
$\left(3^{7} \cdot 5^{4} \cdot 4\right)-1$ & 5.467 .499 & 23 \\
$\left(3^{11} \cdot 5^{6} \cdot 4\right)-1$ & 11.071 .687 .499 & 34 \\
\hline
\end{tabular}

Neste trabalho, utilizou-se um valor máximo de $p$ correspondente ao tamanho de 34 bits. Evidentemente, tais valores de $p$ são insuficientes para garantir a segurança do protocolo proposto. [Azarderakhsh et al. 2016], [Feo et al. 2014] consideram que o tamanho do valor de $p$ para a segurança de um protocolo criptográfico pós-quântico baseado em isogenias de curvas elípticas seja no mínimo igual a 512 bits. No entanto, isso não invalida a prova de conceito do $\left(\begin{array}{l}2 \\ 1\end{array}\right)$-SIOT. Além disso, considerando que o tipo de operação mais onerosa no nosso protocolo é o cálculo de isogenias e, assim, ao compará-lo com os dois protocolos OT pós-quânticos de [Vitse 2019], verificou-se que o $\left(\begin{array}{l}2 \\ 1\end{array}\right)$-SIOT requer menos operações desse tipo.

\section{Conclusão}

Neste artigo, foi apresentada uma proposta de um protocolo pós-quântico chamado $\left(\begin{array}{l}2 \\ 1\end{array}\right)$ SIOT cuja segurança é fundamentada na dificuldade de um adversário calcular isogenias entre curvas elípticas supersingulares e na inspiração da relativa simplicidade do protocolo OT de[Chou and Orlandi 2015] para assegurar a privacidade entre o remetente e destinatário. No que se refere a essa privacidade, foi importante elaborar um teorema compatibilizando uma definição de privacidade OT de [Hazay and Lindell. 2010] com problemas computacionais de isogenias entre curvas elípticas supersingulares de [Feo et al. 2014], considerando um cenário hipotético entre um remetente desonesto e um destinatário honesto, e vice-versa. Por fim, uma análise algébrica com o emparelhamento de Weil delineou certas condições necessárias para que não houvesse violações de segurança e privacidade no protocolo proposto.

\section{Referências}

Azarderakhsh, R., Koziel, B., Jalali, A., Kermani, M. M., and Jao, D. (2016). Neonsidh: Efficient implementation of supersingular isogeny diffe - hellman key exchange protocol on arm. Cryptology ePrint Archive, Report 2016/669.

Barak, B. (2007). Oblivious transfer and private information retrieval. https://www.cs.princeton.edu/courses/archive/fall07/cos433/lec19.pdf. 
Bennett, C. H., Brassard, G., Crépeau, C., and Skubiszewska, M.-H. (1992). Pratical quantum oblivious transfer. In Feigenbaum, J., editor, Advances in Cryptology CRYPTO '91: Proceedings, pages 351-366, Berlin, Heidelberg. Springer Berlin Heidelberg.

Chou, T. and Orlandi, C. (2015). The simplest protocol for oblivious transfer. In Lauter, K. and Rodríguez-Henríquez, F., editors, Progress in Cryptology - LATINCRYPT 2015: 4th International Conference on Cryptology and Information Security in Latin America, Guadalajara, Mexico, August 23-26, 2015, Proceedings. Springer International Publishing.

Crepeau, C. and Kilian, J. (1988). Achieving oblivious transfer using weakened security assumptions. In 29th Annual Symposium on Foundations of Computer Science, pages $42-52$.

Even, S., Goldreich, O., and Lempel, A. (1985). A randomized protocol for signing contracts. ACM, 28(6):637-647.

Feo, L. D., Jao, D., and Plût, J. (2014). Towards quantum-resistant cryptosystems from supersingular elliptic curve isogenies. Journal of Mathematical Cryptology, 8(3):209_ 247.

Galbraith., S. D. (2012). Mathematics of public key cryptography. Cambridge University Press, Cambridge.

Hazay, C. and Lindell., Y. (2010). Efficient Secure Two - Party Protocols - Techniques and Constructions. Springer Berlin Heidelberg.

Hoffstein, J., Pipher, J., and Silverman, J. H. (2014). An introduction to mathematical cryptography. Undergraduate Texts in Mathematics. Springer, New York, second edition edition.

Kalai, Y. T. (2005). Smooth projective hashing and two-message oblivious transfer. Advances in Cryptology - EUROCRYPT 2005., 3494.

Kazmi, R. A. (2015). Cryptography from Post-quantum Assumptions. McGill theses. McGill University Libraries.

Rabin, M. O. (1981). How to exchange secrets with oblivious transfer. Cryptology ePrint Archive, Report 2005/187.

Rogaway, P. (2004). On the role of definitions in and beyond cryptography. Springer and Berlin and Heidelberg, 3321.

Silvermann, J. H. (2009). The arithmetic of elliptic curves, volume 106 of Graduate Texts in Mathematics. Springer, Dordrecht, second. edition.

Vitse, V. (2019). Simple oblivious transfer protocols compatible with kummer and supersingular isogenies. hal-01981552.

Wagner, D. (2016). Technical perspective: Fairness and the coin flip. Communications of the ACM., 59(4):75.

Washington., L. C. (2008). Elliptic curves - Number Theory and Cryptography. Taylor \& Francis Group. LLC, second edition. edition. 